\title{
Flow of a Thixotropic Bingham Fluid over a Cylinder in Stationary and Non-Stationary Regimes
}

\author{
C. M. Bui ${ }^{1}$ and T. X. Ho ${ }^{1,2 \dagger}$ \\ ${ }^{1}$ Faculty of Engineering, Vietnamese-German University Le Lai Street, Hoa Phu Ward, Thu Dau Mot City, \\ Binh Duong Province, Vietnam \\ ${ }^{2}$ Institute for Computational Science and Technology, SBI Building Quang Trung Software City, Tan Chanh \\ Hiep Ward, District 12, Ho Chi Minh City, Vietnam \\ †Corresponding Author Email: thinh.hx@vgu.edu.vn
}

(Received November 3, 2019; accepted February 8, 2020)

\begin{abstract}
Flow of a thixotropic Bingham liquid over a cylinder is investigated in this work. Thixotropy is a rheological characteristic associated with the microstructure of the material, which can be broken and recovered during a flowing process. Various flow characteristics such as the flow morphology, the unyielded/yielded zones, the microstructural state, and the hydrodynamic forces acting on the cylinder are analyzed at $\mathrm{Re}=20-100$ and $\mathrm{Bn}$ $=0.5-10$. Comparison with Newtonian and non-thixotropic Bingham fluids are also carried out and discussed. Results show that, within these conditions, the flow spans from a stationary regime with or without separation to a non-stationary laminar one with von-Karman-type vortex shedding. In addition, no near-field unyielded zones are observed at $\mathrm{Re}=100$ and $\mathrm{Bn}=0.5$ and 1 , probably due to the unsteady nature of the flow. At conditions where static unyielded zones exist, the wall shear stress normalized by the yield stress is minimum within these structures.
\end{abstract}

Keywords: Bingham; Thixotropy; Computational fluid dynamics.

\section{INTRODUCTION}

Non-Newtonian liquids are frequently encountered in nature (e.g., mud and lava flows) and engineering (e.g., sediment suspension, fresh cement/concrete, fuel oil, polymer, food, and drug). They can possess yield stress and thixotropic characteristics. A yield stress liquid flows only when an applied shear stress $\tau$ is greater than a threshold value $\tau_{0}$, i.e., the yield stress, otherwise behaves like a solid. Thixotropy is a rheological property associated with the materials' microstructure, which can evolve during a flowing process. The microstructural evolution is assumed to consist of two opposite processes: breakdown and recovery. The former makes the materials more fluid, meanwhile the latter makes them more solid. Specifically, their yield stress and hence apparent viscosity change with the microstructure status. A flow of such materials is much more complex than that of Newtonian ones. Typically, there can exist zones within which $\tau \leq \tau_{0}$, and fluid particles do not move relatively with each other but stick together and become rigid or solid-like zones, i.e. so-called unyielded zones. They can either stick to a solid wall or be suspended in the flow field. Other zones, i.e., within which $\tau>\tau_{0}$, are called yielded ones. A boundary between the yieded and unyielded zones is called yield boundary, line or surface.

Although these liquids have many important applications, their flow behaviors have not been well understood. This work is, therefore, to carry out a detailed investigation of a thixotropic flow over a circular cylinder. This setting is an idealization of problems encountered in many engineering systems involving fluid-solid interactions such as a $\mathrm{mud} /$ sediment flow over a pipeline in an event of a submarine landslide, flow of fresh cement/concrete through reinforcement, chemical manufacturing, and food processing.

Tokpavi et al. (2008) investigated viscoplastic fluid, a Bingham type, at high Oldroyd numbers past a cylinder using a numerical approach. High Od means strong plastic effects over viscous effects. It is noted that the flow was very slow or in the limit of zero Reynolds number, i.e., $\mathrm{Re}=\mathrm{O}(0)$, inertia effect was therefore negligible. Unyielded zones were observed and classified; among them, two stuck to the cylinder at upstream and downstream stagnation points, i.e., so-called static rigid zones. The size and shape of the unyielded zones were reported to depend on Od, however, they were asymptotic at high $\mathrm{Od}$, i.e., $\mathrm{Od}=$ 
$2 \times 10^{5}$. In their subsequent work (Tokpavi et al. 2009), velocity measurement using PIV technique was carried out. The fluid was a shear-thinning viscoplastic one, and the flow was still in the limit of zero Re. Asymmetry between the upstream and down-stream was reported, that is, a flow recirculation zone was observed at the front stagnation point, whereas at the rear point was a rigid zone. Furthermore, Ribeiro et al. (2014) explored streamline pattern and velocity field of a flow past a cylinder confined in a rectangular duct. The fluids were viscoelastic including shear-thinning and Boger ones (aqueous solutions of polyacrylamide without or with glycerin), for which Carreau and Oldroyd-B models were, respectively, employed. The flow was in the range from creeping to elasticinduced time-dependent conditions, e.g., $\mathrm{Re}=$ $0.008-3.2$, and Deborah number De $=0.01-0.14$. The flow of Boger fluids was reported to be symmetric both the upstream and downstream of the cylinder.

At more elevated Re, Mossaz et al. (2010), Mossaz et al. (2012b) and Mossaz et al. (2012a) performed intensive studies on a viscoplastic flow past a circular cylinder using both numerical and experimental approaches. The fluid was of a Herschel-Bulkley type, and Re was up to 100 . Various flow characteristics such as recirculation zones, unyielded/yielded zones and streamline patterns were analyzed. Drag coefficient on the cylinder was in addition reported. Neither a rigid nor a recirculation zone was observed at the upstream stagnation point. At the downstream was either a static zone or a recirculation bubble depending on Re and/or Od. Despite of relatively high Re, since Od was also high, the flow was still in a stationary laminar regime with a pair of symmetrical recirculation zones (inside the bubble) formed behind the cylinder. The onset of periodic nonstationary flow with vortex shedding was identified and reported to depend on a combination of Re and $\mathrm{Od}$; in general, the higher Od, the higher Re.

Other relevant works include, among others, Pantokratoras (2018) and Wang et al. (2019). The former studied a flow induced by a rotating sphere in a power-law fluid. Torque was evaluated at $\mathrm{Re}=$ $0.01-10000$ based on the rotational speed. It was shown that at $\operatorname{Re} \leq 1$, the torque was independent of $\mathrm{Re}$ in shear-thinning fluids; and at high $\mathrm{Re}$ it increased with increasing the power-law index. The latter investigated the rising of a bubble in a Herschel-Bulkley fluid at low Re using experimental and numerical approaches. A model for predicting the rising velocity was proposed, which was stated to be of high accuracy at $\operatorname{Re}<0.4$.

It is noted that in all of the aforementioned works the fluid was non-thixotropic though it possessed either viscoplastic, viscoelastic or power-law rheological behaviors. In the current paper, focus is on the flow of a thixotropic fluid over a cylinder. In case vortices are present in such a flow, they can give rise to the breakdown rate of the microstructure, making it different from that of a non-thixotropic one. The thixotropy was well described in Mewis and Wagner
(2009) and Barnes (1997); and a simple model for it was proposed by Moore (1959). It was employed by Derksen (2011) for a thixotropic flow in a mixing tank. And most recently, Syrakos et al. (2017) utilized it to investigate a stationary laminar flow over a cylinder at $\mathrm{Re}=45$ and $\mathrm{Bn}=0.5$ and 5 . Focus was on effects of the breakdown and recovery controlling parameters on the flow structure.

In this work, the flow regime would span from a stationary one with or without separation to a nonstationary one with vortex shedding behind the cylinder. Indeed, simulations at $\mathrm{Re}=20-100$ and $\mathrm{Bn}$ $=0.5-10$ are realized. Effects of $\mathrm{Bn}$ on the vortex structure are investigated. Other aspects of the flow such as the yielded/unyielded zones, the streamline patterns and hydrodynamic forces acting on the cylinder are analyzed.

\section{THEORY BACKGROUND}

\subsection{Governing Equations}

The governing equations include a continuity and a momentum one, respectively, as follows,

$\nabla \cdot \boldsymbol{u}=0$

$\rho\left(\frac{\partial u}{\partial t}+\boldsymbol{u} \cdot \nabla \boldsymbol{u}\right)=\rho \boldsymbol{f}+\nabla \cdot \boldsymbol{\sigma}$

where $\boldsymbol{u}$ and $\boldsymbol{f}$ are the velocity and body force vectors, respectively; $\rho$ is the fluid density; and $\boldsymbol{\sigma}=-\mathrm{p} \boldsymbol{I}+\boldsymbol{\tau}$ is the total stress tensor with p being pressure, $\boldsymbol{I}$ the unit tensor and $\tau$ the deviatoric stress tensor. It is defined as $\tau=\mu \dot{\gamma}$ for Newtonian fluid; $\dot{\gamma}$ is the deformation rate tensor with $\dot{\gamma}_{i j}=\left(\partial u_{j} / \partial x_{i}+\partial u_{i} / \partial x_{j}\right)$. However, for Herschel-Bulkley (HB) fluid, it is defined as follows,

$\begin{cases}\tau=\left(K \dot{\gamma}^{n-1}+\frac{\tau_{0}}{\dot{\gamma}}\right) \dot{\gamma} & \text { if } \tau>\tau_{0} \\ \dot{\gamma}=0 & \text { if } \tau \leq \tau_{0}\end{cases}$

Here, $\tau_{0}$ is the yield stress; $K$ is the consistency and $\dot{\gamma}$ is the strain rate tensor's magnitude. $n$ is the power index controlling the rate of change of fluid viscosity; for Bingham fluid, $n=1$. This model has a discontinuity at the transition between the solid-like and fluid-like behaviors. To tackle this, Papanastasiou's regularization technique (Papanastasiou 1987) is employed:

$\boldsymbol{\tau}=\left(K \dot{\gamma}^{n-1}+\frac{\tau_{0}[1-\exp (-m \dot{\gamma})]}{\dot{\gamma}}\right) \dot{\gamma}$

where $m$ is the regularization parameter. When $m \rightarrow \infty$, Eq. (4) approaches Eq. (3) for an ideal Bingham fluid; and for which the strain rate is absolutely zero when $\tau \leq \tau_{0}$. However, as everything deforms even when $\tau \leq \tau_{0}$, we choose $m=40000$ corresponding to a finite strain rate, which is $\dot{\gamma}_{c}=$ $0.0001 \mathrm{~s}^{-1}$, i.e. the so-called critical shear rate. This fluid is similar to Carbopol gels used in Mossaz et al. (2012a), making it possible for a validation. Detailed information about the determination of $m$ can be referred to Bui and Ho (2019).

Reynolds and Oldroyd numbers are defined as, respectively, 
(a)

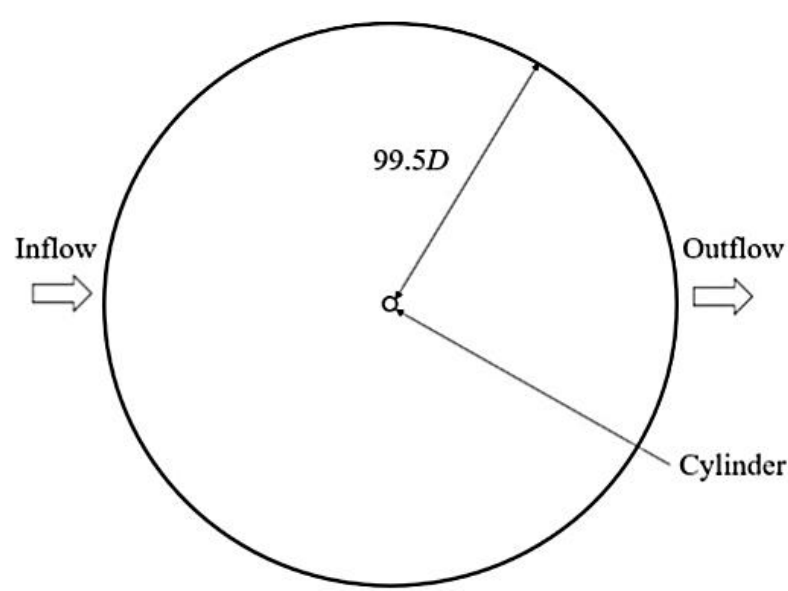

(b)
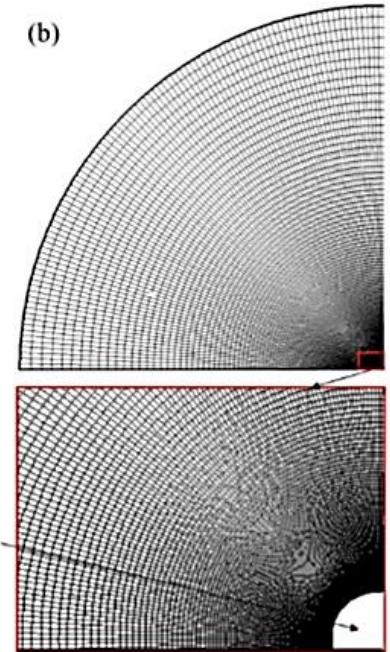

Fig. 1. Computational domain (a) and mesh consisting of 92000 elements (b).

$\operatorname{Re}=\frac{\rho u_{\infty}^{2-n} D^{n}}{K}$

$\mathrm{Od}=\frac{\tau_{y} D^{n}}{K u_{\infty}^{n}}$

with $D$ being the cylinder's diameter, $u_{\infty}$ the far field velocity, and $\tau_{y}$ the yield stress when the microstructure is fully built up. When $n=1$, the liquid is a Bingham one for which the Bingham number is employed as

$\mathrm{Bn}=\frac{\tau_{y} D}{K u_{\infty}}$

\subsection{Thixotropy}

Thixotropy is a complex viscoplastic property of a material associated with the evolution of its microstructure during a flowing process. Two counter thixotropic processes, i.e., the recovery and the breakdown ones, are assumed to take place simultaneously. The recovery process is attributed to in-flow phenomena, e.g., collision or orthokinetic coagulation, and Brownian motion effect. The breakdown is associated with a reduction in the connection among molecules/particles due to shear stress.

In addition, the thixotropic characteristic can be associated with a system of flocculated particles, in which, floc size represents the build-up ability of the microstructure. When the recovery process takes a predominant role in the structural transition, e.g., at rest or when small shear stress is applied, the development of flocs is expected. This creates strong links among molecules or particles and large attractive force between them, which then result in a high viscosity and all these lead to low flowability of the material. In contrast, a fast breakdown mechanism would strongly reduce the floc size, and the molecules' bonds in the microstructure would be fractured. These decrease the fluid viscosity and make the material more fluid.

The microstructural state is represented by a dimensionless structural parameter, $\lambda$, which varies from 0 (completely broken/unstructured state) to 1 (fully structured state). Its evolution is modeled by a kinetic equation (Moore 1959):

$\frac{\partial \lambda}{\partial t}+\boldsymbol{u} \cdot \nabla \lambda=\alpha(1-\lambda)-\beta \lambda \dot{\gamma}$

Here, $\alpha$ and $\beta$ are the recovery and breakdown parameters, respectively. In this work, $\alpha=0.05$ and $\beta=$ 0.05 though their exact values need to be determined by experiments. The first and second terms on the RHS of Eq. (8) represent the recovery and breakdown processes, respectively. At equilibrium, they balance each other. The yield stress is determined as $\tau_{0}=\lambda \tau_{\mathrm{y}}$ with $\tau_{\mathrm{y}}$ being evaluated at $\lambda=$ 1. When $\lambda=0, \tau_{0}=0$, and the fluid becomes Newtonian. Therefore, in a flow field, $\tau_{0}$ can vary from 0 to $\tau_{y}$, and distribute non-uniformly.

\section{IMPLEMENTATION AND VALIDATION}

Numerical simulations are performed using Ansys Fluent 14.5. Two User-Defined Functions (UDF) are developed to incorporate Eqs. (4) and (8). The problem is two-dimensional (2D) as the cylinder is assumed to be infinitely long and three-dimensional (3D) aspects of the flow are assumed to be negligible. Figure 1 shows a circular computational domain employed in this work. The domain's diameter is $D_{\infty}$ with $D_{\infty} / D=200$, which is supposed to be sufficient (Mossaz et al. 2010). A structured mesh consisting of 92000 elements with high resolution near the cylinder is utilized. Mesh convergence study has been carried out and presented elsewhere (Bui and Ho 2019). The flow is laminar; and non-slip boundary condition is applied to the cylinder's wall.

For the validation purpose, a non-thixotropic Herschel-Bulkley liquid and a thixotropic Bingham one are employed here. Simulation of the nonthixotropic one at $\mathrm{Re}=35$ and $\mathrm{Od}=0.34$ is realized; results for the streamlines and unyielded zones are shown in Fig. 2, which are found to agree well with those obtained both numerically and experimentally by Mossaz et al. (2012a). The thixotropic flow is 


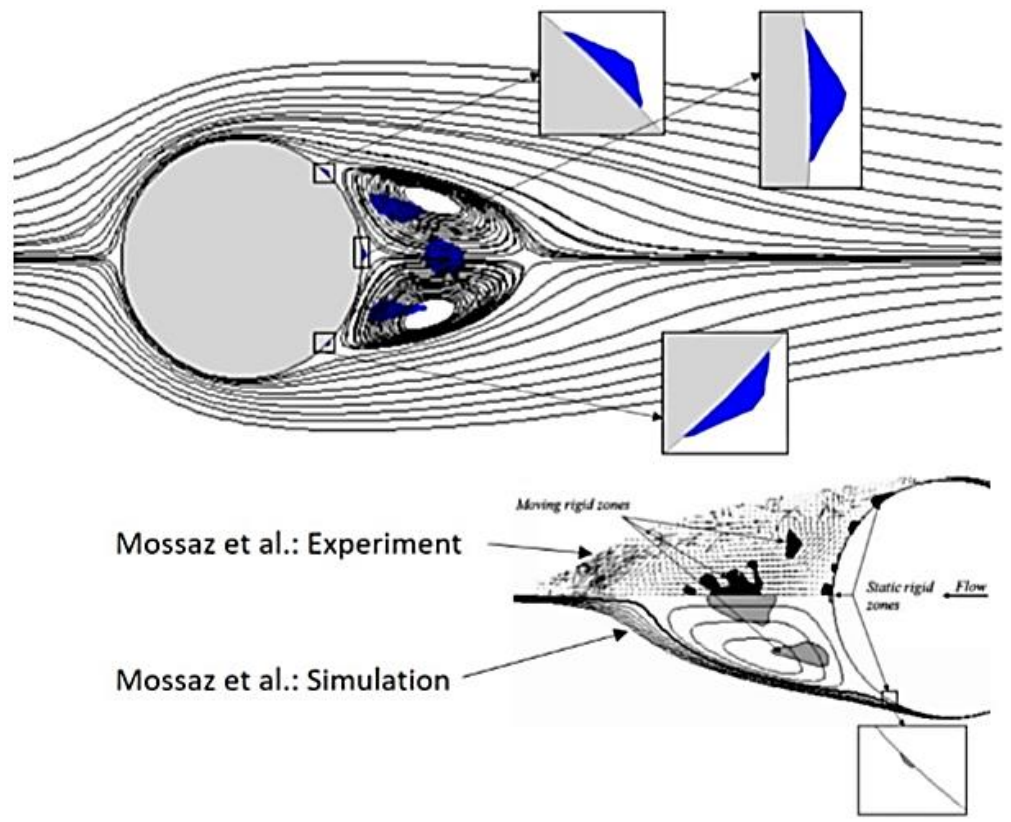

Fig. 2. Streamline pattern and unyielded zones of a $\mathrm{HB}$ fluid at $\mathrm{Re}=35$ and $\mathrm{Od}=0.34$. Top: current simulation; Bottom: results of Mossaz et al. (2012a) (License number of 4732381237460).
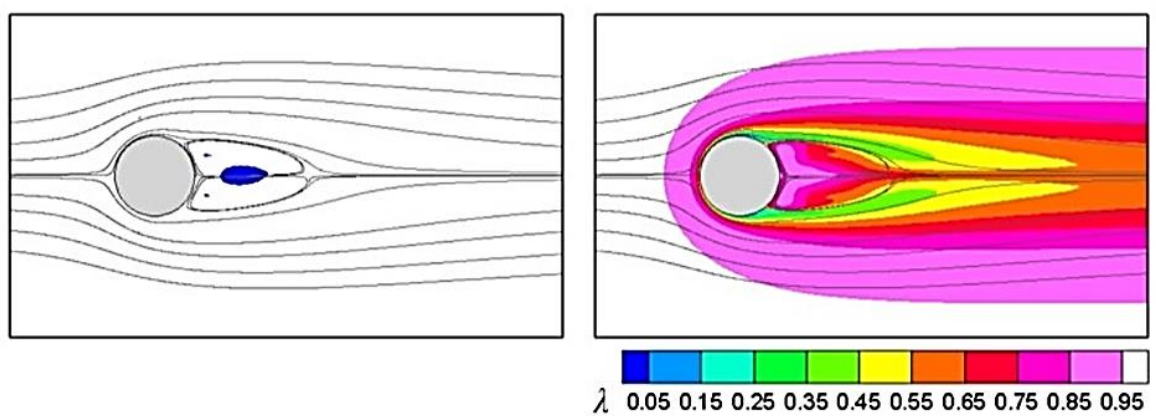

Fig. 3. Unyielded zones ( $\mathrm{L})$ and distribution of $\lambda(\mathrm{R})$ at $\mathrm{Re}=45, \mathrm{Bn}=0.5$. Streamlines are shown as well.

realized at $\mathrm{Re}=45$ and $\mathrm{Bn}=0.5$; results for the streamlines, the near-field unyielded zones, and the distribution of the structural parameters are illustrated in Fig. 3. Good agreement with those obtained numerically by Syrakos et al. (2017) is achieved.

\section{RESULTS AND DISCUSSION}

In this work, $\mathrm{Bn}$ is in the range of $0.5-10$, and $\mathrm{Re}$ in the range of 20-100. Regarding the latter, focus is paid on cases of $\operatorname{Re}=60$ and 100 . It should be noted that at these Re, a flow of Newtonian fluids would be in an unsteady laminar regime with vortex shedding behind the cylinder (Tritton 1959; Williamson 1996), however, it is not necessarily the case for Bingham ones, which are presented in this section.

\subsection{Stationary flow at $\operatorname{Re}=60$}

Results for the streamline pattern and the unyielded zones of a thixotropic fluid at $\operatorname{Re}=60$ and $\mathrm{Bn}=0.5$ are presented in Fig. 4. Those of Newtonian and Bingham non-thixotropic $(\mathrm{Bn}=0.5)$ ones at the same $\mathrm{Re}$ are also shown therein. The flow of these fluids can be considered equivalent, and the term of equivalent flow is used in the following part of the paper. It is obvious that the Newtonian flow is nonstationary with periodic vortex shedding behind the cylinder. The Bingham thixotropic and nonthixotropic ones are, however, found to be stationary with a symmetrical recirculation bubble instead. The bubble is larger for the thixotropic fluid than for the non-thixotropic one. This can be attributed to the fact that, compared to the latter, the microstructure of the former can be broken down, making it less structured and more Newtonian alike, especially in the cylinder's wake. Away from the cylinder where strain rate is small, viscoplastic effects stabilize the flows.

The unyielded zones are defined by $\tau \leq \tau_{\mathrm{y}}$. They are categorized into far-field and near-field ones; among the latter, those that stick to the cylinder are called 

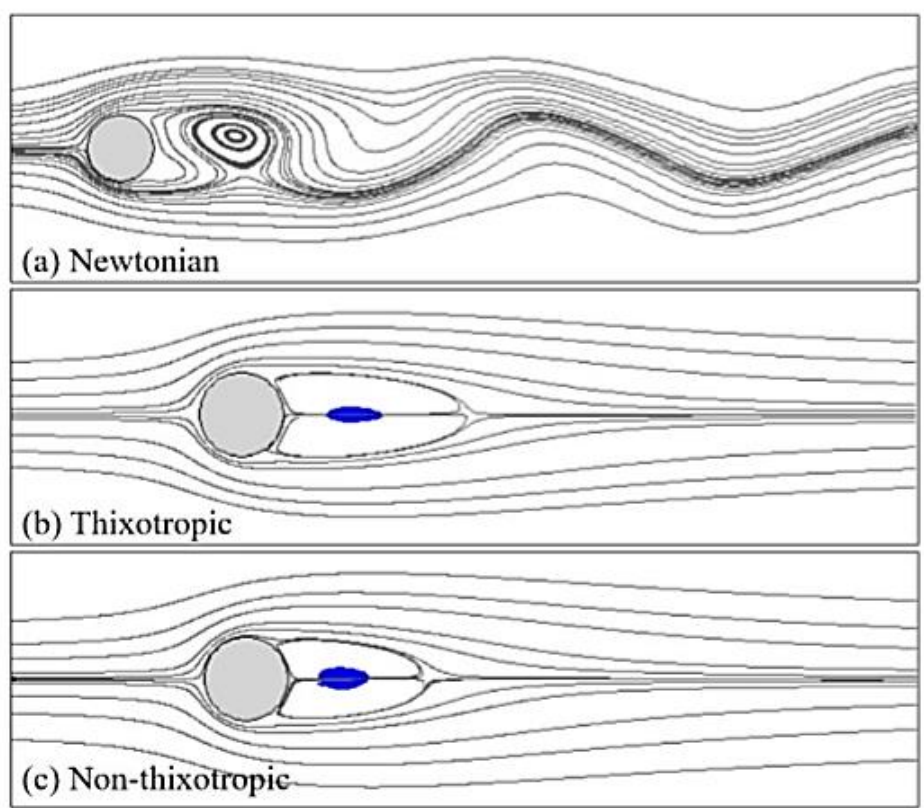

Fig. 4. Streamline pattern and unyielded zones of Newtonain (a), thixotropic Bingham (b) and nonthixotropic (c) fluids at $\operatorname{Re}=60$.

static zones, others called suspended or moving ones. As thixotropy is a time-dependent rheological characteristic, the unyielded/yielded zones and structural state can change with time. Figure 5 shows snapshots of the near-field unyielded zones and the distribution of $\lambda$ at several time points. It is found that the flow reaches equilibrium at $t \sim 0.375 t_{\infty}$ with $t_{\infty}=D_{\infty} / u_{\infty}$, equivalent to $t \sim 75 t_{D}$ with $t_{D}=D / u_{\infty}$. Figure 6 facilitates a closer view of $\lambda$ at equilibrium. It is obvious that $\lambda$ is great, i.e., $\lambda \approx 1$, in static rigid zones as within which the strain rate is almost vanished and thus the recovery process dominates over the breakdown one. It is smaller near the end of the recirculation bubble where the strain rate is supposed to be large or the material is to be sheared and hence broken. Further downstream, however, $\lambda$ increases as the microstructure is recovered. Recall that the yield boundary, which is $\sim 30 \mathrm{D}$ behind the cylinder here, separates the yielded and unyielded zones, beyond it, $\dot{\gamma}<\dot{\gamma}_{c}=0.0001 \mathrm{~s}^{-1}$ and the fluid is unyielded; however, the recovery process continues farther until a considerable distance. The breakdown is seen to take place mostly in the wake, and the fluid remains fully structured in a large area in front and on lateral sides of the cylinder, even within the yield boundary.

Figure 7 shows comparison of the near-field unyielded zones between the thixotropic and nonthixotropic flows. It is noticed that less and smaller rigid zones are formed in the former than in the latter. In addition to this, the location of the static rigid zones on the lateral sides of the cylinder implies that the flow separation occurs further upstream in the thixotropic flow; this agrees well with the size of the recirculation bubble, i.e., larger in the thixotropic (Fig. 4b) than in the non-thixotropic one (Fig. 4c).

It is noteworthy that $\mathrm{Bn}$ has an opposite effect with
Re. The fluid would be more structured, and more and/or larger unyielded zones would be formed at higher $\mathrm{Bn}$. Results for the unyielded zones and the distribution of $\lambda$ at $\operatorname{Re}=60$ and $\mathrm{Bn}=0.5,1,2$ and 5 are presented in Fig. 8. Flow separation is seen to take place forming a symmetrical recirculation bubble at $\mathrm{Bn} \leq 1$. As $\mathrm{Bn}$ increases, the bubble becomes smaller, and at the same time, more rigid zones are formed and/or become bigger inside the bubble, e.g., at $\mathrm{Bn}=2$. They approach the cylinder, combine with the existing static zones and eventually become a single large one, e.g., at $\mathrm{Bn}=5$, within which $\lambda \approx 1$. This static zone can be considered as an extended cylinder; and the fluid flows around the cylinder-rigid combined structure.

\subsection{Non-Stationary flow at $\operatorname{Re}=100$}

Simulations of the flow at $\mathrm{Bn}=0.5$ and various values of $\operatorname{Re}$, i.e., $\operatorname{Re}=20,45,60$, and 100 are performed. Results for the vorticity magnitude are shown in Fig. 9. It is evident that the flow is stationary at $\mathrm{Re} \leq 60$, and non-stationary only at $\mathrm{Re}$ $=100$ with vortex shedding behind the cylinder.

Figure 10 shows results for the distribution of $\lambda$ at $\operatorname{Re}$ $=100$ and $\mathrm{Bn}=0.5$. No static rigid zones are observed though $\lambda$ is relatively great, i.e., up to 0.85 , in some regions on the cylinder. Two moving rigid zones are found on the lateral sides of the wake, far from the wake, and hence are supposed to have little influence on it. Compared to the flow at $\mathrm{Re}=$ 60 and $\mathrm{Bn}=0.5$ presented above, the unstructured wake here is thicker, and the yield boundary is considerably longer, i.e., $\sim 55 D$ behind the cylinder as compared to $\sim 30 \mathrm{D}$.

Additionally, results for the streamline pattern and the near-field rigid zones are shown in Fig. 11, and those for the distribution of $\lambda$ and the vorticity 

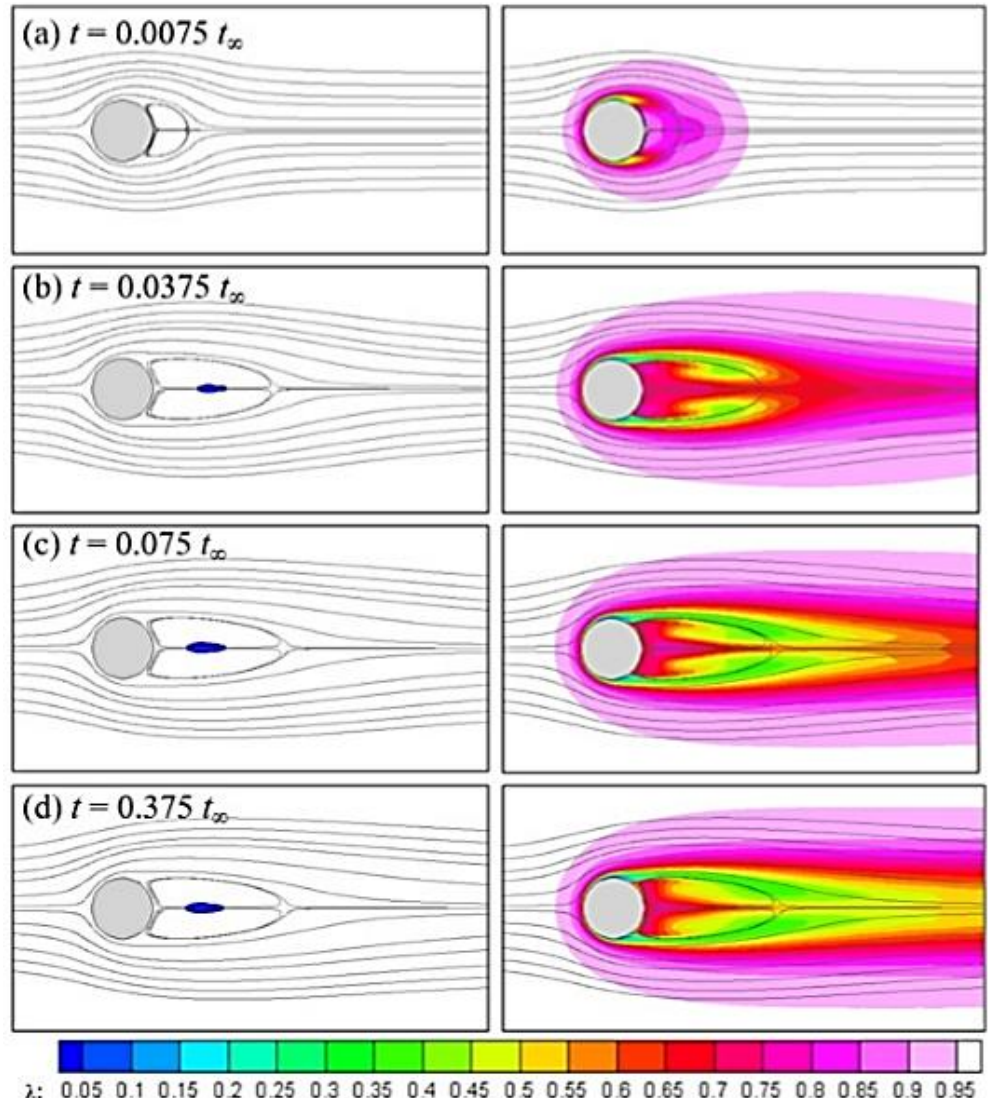

Fig. 5. Snapshots of $\lambda(\mathrm{R})$ and the near-field un-yielded zones $(\mathrm{L})$ at different time points; $\mathrm{Re}=60$ and Bn $=0.5$; streamline patterns are shown on both sides.

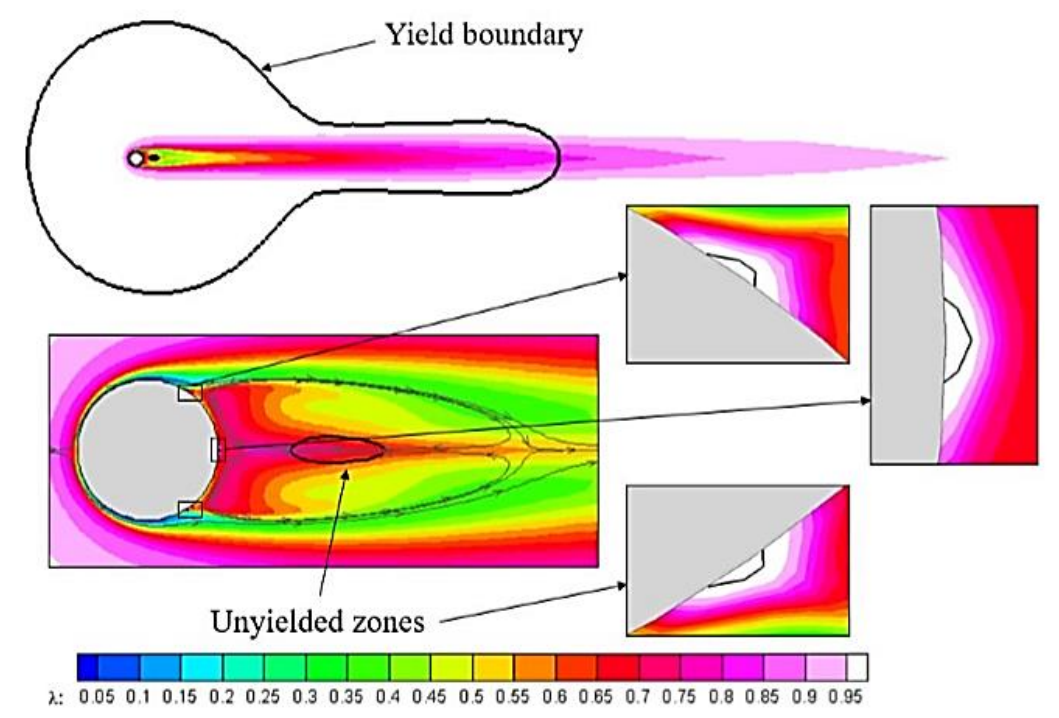

Fig. 6. Distribution of $\lambda$ in the yielded and unyielded zones at $\operatorname{Re}=60$ and $B n=0.5$.

magnitude in Fig. 12. The flow is well in the nonstationary regime with periodic vortex shedding behind the cylinder at $\mathrm{Bn} \leq 1$. At $\mathrm{Bn}=2$, the flow is still non-stationary, however, the symmetry is no longer present, and two recirculation bubbles of different size alternately change their position. It becomes stationary at greater $\mathrm{Bn}$, i.e., $\mathrm{Bn}=5$ and 10 .
Flow separation takes place at $\mathrm{Bn}=5$, forming a pair of symmetrical recirculation zones; however, it does not take place at $\mathrm{Bn}=10$, and a large static zone is instead observed at the downstream stagnation point. It is noticed that, as Bn increases, the unstructured wake becomes narrower, and more unyielded zones are formed and become larger. Moreover, in the non- 


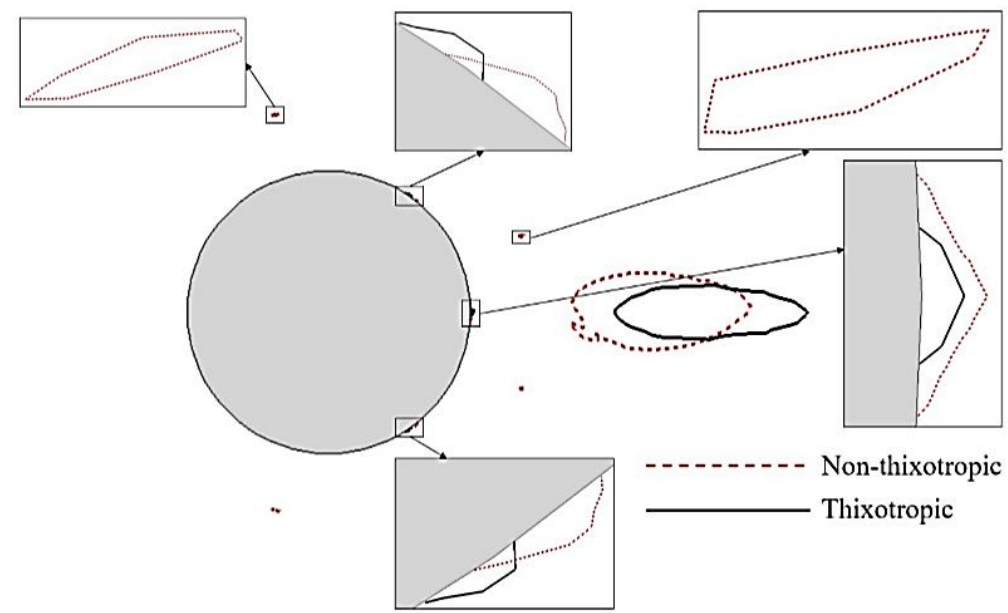

Fig. 7. Comparison of the near-field unyielded zones between the thixotropic and non-thixotropic Bingham flows at $\operatorname{Re}=60$ and $B n=0.5$.

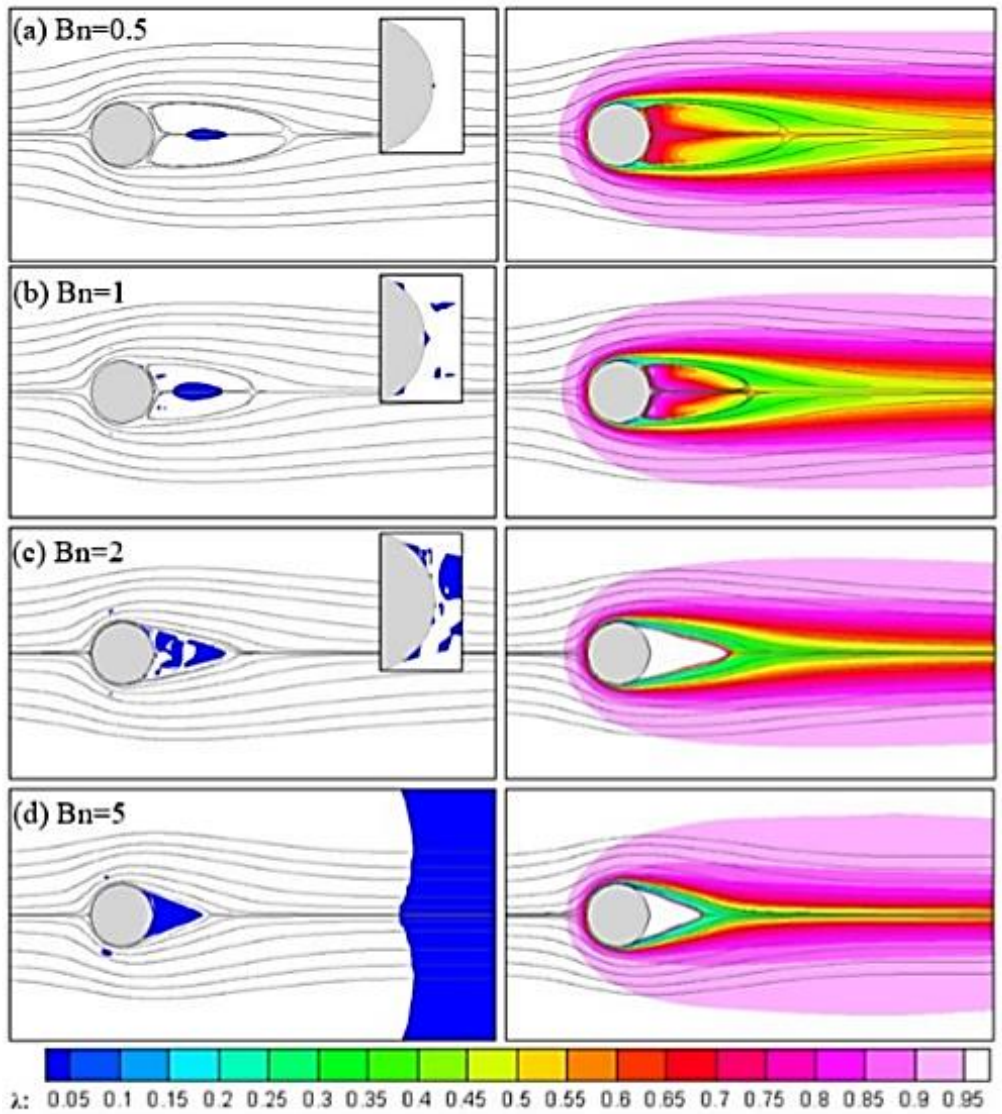

Fig. 8. Unyielded zones ( $\mathrm{L})$ and distribution of $\lambda(\mathrm{R})$ at $\mathrm{Re}=60$ and several values of $\mathrm{Bn}$. Streamlines are shown on both sides. The color bar is for $\lambda$ only.

stationary flow the fluid structure inside the vortices is considerably broken resulting in a low structural parameter, i.e., $\lambda \sim 0.3-0.5$. On the contrary, in the stationary flow the material is well structured with $\lambda$ $\rightarrow 1$ in a large part of the re-circulation bubble and/or the static zone. More time may be required for the structure in the entire static zone to be fully recovered, i.e., at $\mathrm{Bn}=10$.

\subsection{Hydrodynamic Forces}

In this subsection, results for the drag coefficient, $C_{d}$ $=2 F_{d} /\left(\rho u_{\infty}^{2} A\right)$, the pressure coefficient, $C_{p}=2(p-$ $\left.p_{\infty}\right) / \rho u_{\infty}^{2}$, and the wall shear stress, $\tau_{\mathrm{w}}$, acting on the cylinder's surface are analyzed; $F_{d}$ is the drag force, $A$ the reference area and $p_{\infty}$ the reference pressure.

Figure 13 presents $C_{d}$ of the thixotropic Bingham 

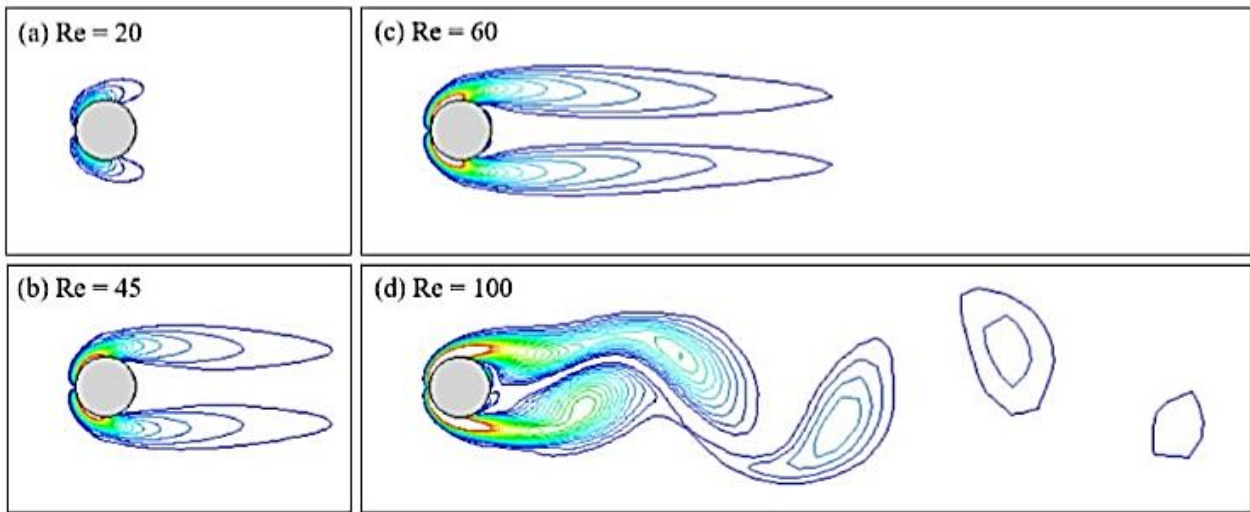

Fig. 9. Contours of the vorticity magnitude of the thixotropic flow at different values of Re, and $B n=0.5$.

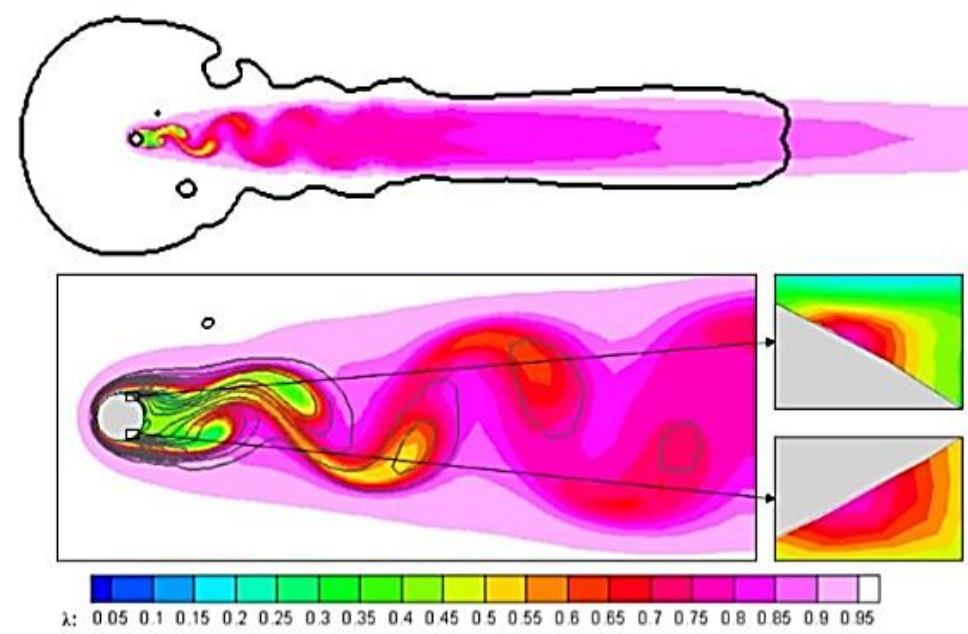

Fig. 10. Distribution of $\lambda$ in the thixotropic flow at $R e=100$ and $B n=0.5$. Two small circles are the moving rigid zones. Contours of the vorticity magnitude are shown as well.
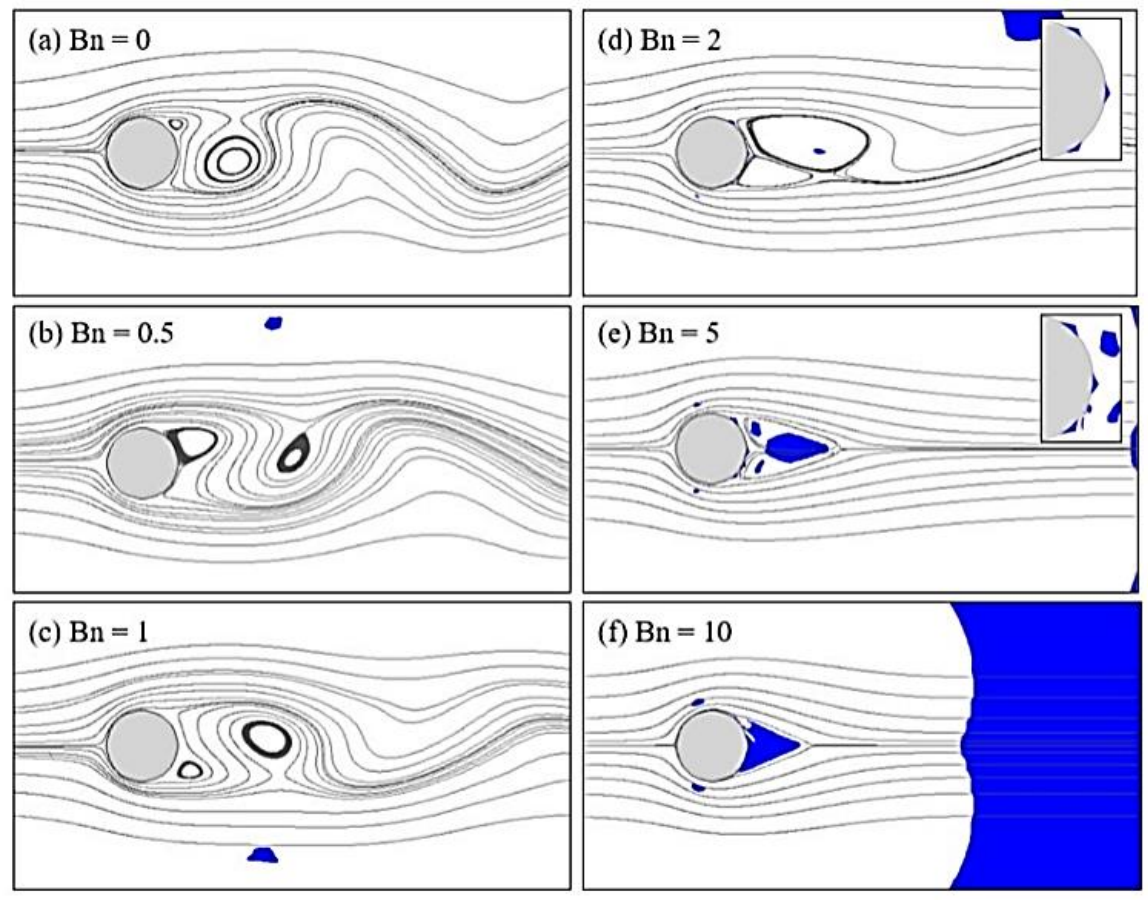

Fig. 11. Streamlines and unyielded zones (shaded areas) at $R e=100$ and $B n=0-10$. The large shaded area in (f) is the far-field unyielded zone. 

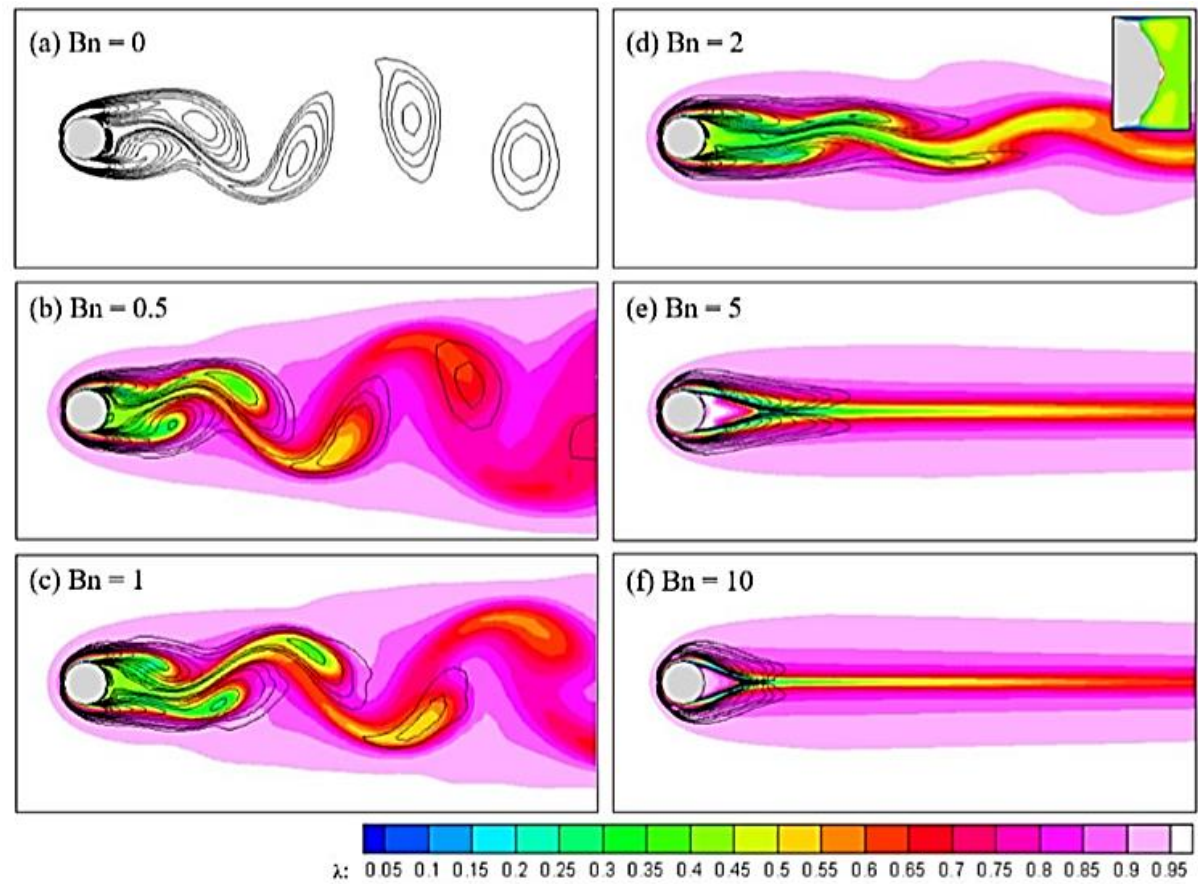

Fig. 12. Distribution of $\lambda$ and contours of the vorticity magnitude at $\operatorname{Re}=100$ and $B n=0-10$.

flow as a function of Re; that of the equivalent flows of non-thixotropic Bingham and Newtonian fluids is also shown therein. $\mathrm{Bn}=0.5$ for both Bingham flows. It is found that $C_{d}$ of the thixotropic flow is smaller than that of the non-thixotropic one at relatively small $\mathrm{Re}$, i.e., $\mathrm{Re}=45$ and 60 . However, the trend is completely reversed at $\mathrm{Re} \geq 100$, where the flow is in the non-stationary regime. $C_{d}$ of the Newtonian fluid is always the smallest. Moreover, as Re increases, $C_{d}$ of the Bingham flows gets closer to that of the Newtonian one; indeed, difference between the three fluids is found to be insignificant at $\operatorname{Re}=150$. This can be due to the fact that the drag force is dictated by the flow near the cylinder, where the Bingham materials are yielded (no unyielded zones are formed) at high Re, and thus both behave like a Newtonian one. The data for $C_{d}$ at $\mathrm{Re}=150$ are from Bui and Ho (2019).

Effects of Bn on $C_{d}$ of the thixotropic flow at $\mathrm{Re}=$ 45, 60 and 100 are illustrated in Fig. 14. It is found that $C_{d}$ increases linearly with $\mathrm{Bn}$ in the range investigated. In addition, at the same $\mathrm{Bn}, C_{d}$ is smaller at a higher Re; this trend is more pronounced as $\mathrm{Bn}$ increases.

Furthermore, Fig. 15 shows the distribution of $C_{p}$ on the cylinder's surface at $\mathrm{Re}=60$ and 100 and various values of $\mathrm{Bn}$; that of Newtonian and inviscid fluids is also presented therein. As can be observed, the $C_{p}$ curves possess two minima on the lateral sides, and a maximum at the front stagnation point. The latter increases significantly as $\mathrm{Bn}$ increases. Some fluctuations in $C_{p}$ exist in the downstream part of the cylinder, which can be due to the formation of the unyielded zones at relatively high $\mathrm{Bn}$, i.e., $\mathrm{Bn}=2$ and 5 for $\mathrm{Re}=60$, and $\mathrm{Bn}=5$ for $\mathrm{Re}=100$. Nevertheless, in general, $C_{p}$ in this part decreases as Bn increases; this trend is more pronounced for $\mathrm{Re}=60$ than for $\operatorname{Re}=100$. As a consequence, the resulting drag coefficient increases with the increasing $\mathrm{Bn}$, more rapidly for $\operatorname{Re}=60$ than for $\operatorname{Re}=100$. This agrees well with the slope of the $C_{d}-\mathrm{Bn}$ curves shown in Fig. 14.

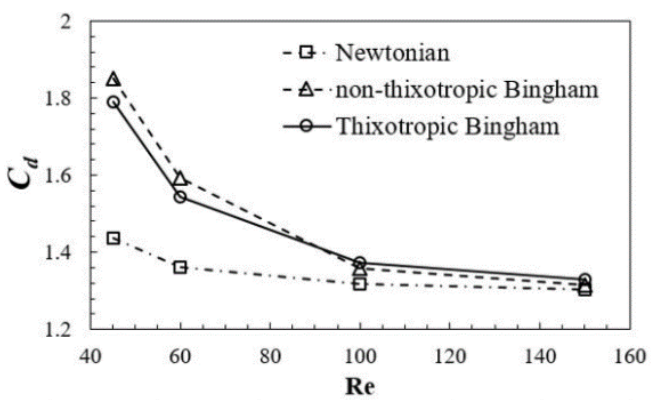

Fig. 13. Drag coefficient versus $\mathrm{Re} ; \mathrm{Bn}=\mathbf{0 . 5}$.

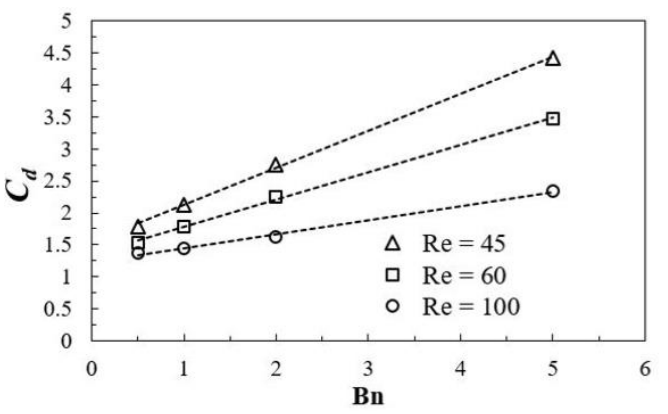

Fig. 14. Drag coefficient of the thixotropic flow as a function of $\mathrm{Bn}$.

Additionally, the position of the minima of the thixotropic flow at $\mathrm{Bn}=0.5$ is found to be almost the 

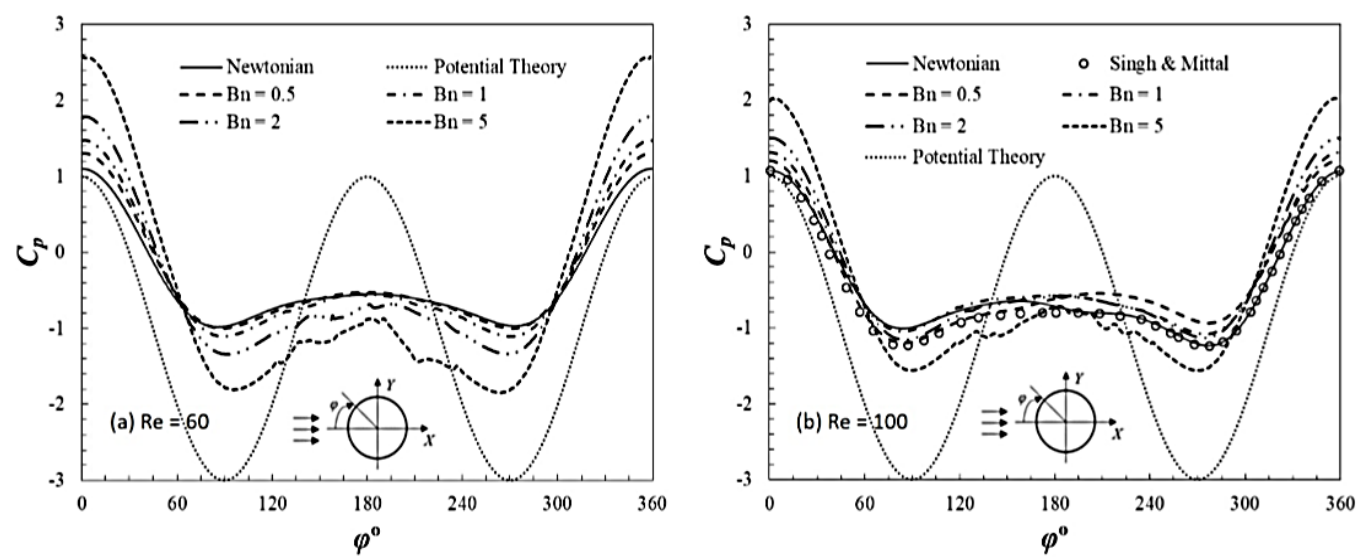

Fig. 15. Distribution of $C_{p}$ on the cylinder's surface at $R e=60$ (a) and 100 (b) and various values of $B n$.
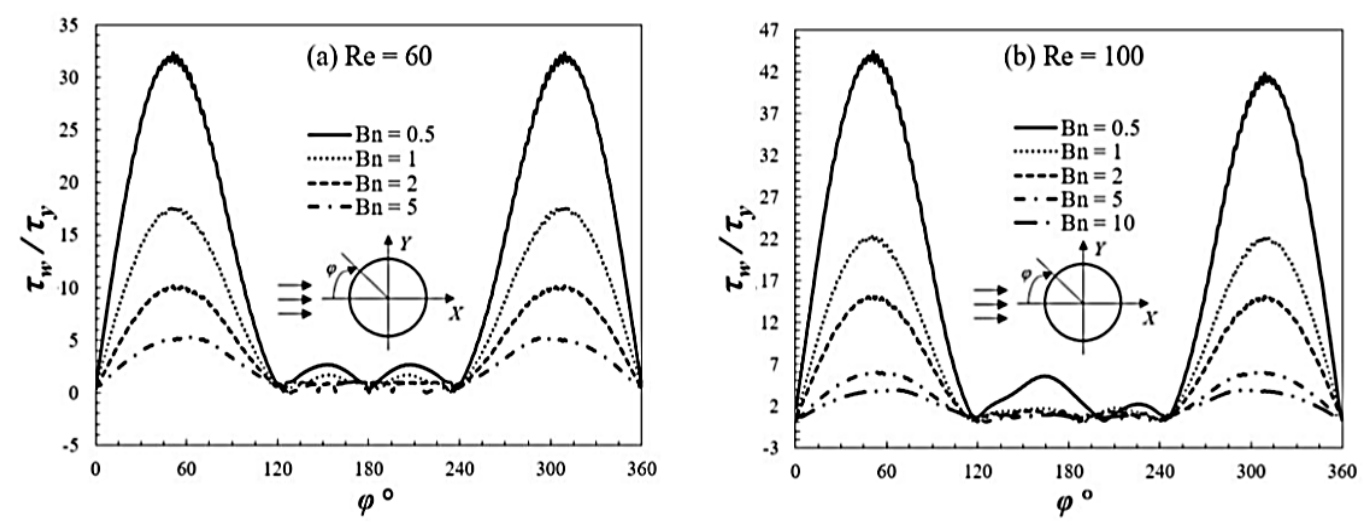

Fig. 16. $\tau_{\mathrm{w}} / \tau_{\mathrm{y}}$ of the thixotropic fluid at $\operatorname{Re}=60$ (a) and 100 (b) and various values of $\mathrm{Bn}$.

same with that of the equivalent Newtonian one. It is at $\varphi=88.05^{\circ}$ and $271.95^{\circ}$ for $\operatorname{Re}=60$, and at $82.58^{\circ}$ and $277.42^{\circ}$ for $\operatorname{Re}=100$. It tends to move slightly downstream as $\mathrm{Bn}$ increases. It is to be mentioned that our results for the minima's position of the Newtonian fluid agree well with those reported by Braza et al. (1986), Park et al. (1998) and Silva et al. (2003). Moreover, the curve for Newtonian fluid at $\mathrm{Re}=100$ is found to fit perfectly with that of Singh and Mittal (2005) on the lower surface of the cylinder. Deviation on the upper surface is probably due to the unsteady nature of the flow. Note that our results shown here are at a time instant, whereas those of Singh and Mittal (2005) are time averaged.

Likewise, results for the wall shear stress normalized by the maximum yield stress, i.e., $\tau_{\mathrm{w}} / \tau_{\mathrm{y}}$, on the cylinder are shown in Fig. 16. In general, the curves of $\tau_{\mathrm{w}} / \tau_{\mathrm{y}}$ are symmetrical with respect to the horizontal axis. Fluctuations are observed on the rear side of the cylinder, i.e., in the region $\varphi \approx 120-240^{\circ}$, at relatively high $\mathrm{Bn}$, i.e., $\mathrm{Bn}=2$ and 5 . This can be attributed to the rigid zones, some of which stay near the cylinder, others attach to it. Figure 17 shows a better view of the fluctuations. As described therein for two cases, i.e., $\mathrm{Bn}=1$ and $\mathrm{Re}=60$ (Fig. 16a), and $\mathrm{Bn}=2$ and $\mathrm{Re}=100$ (Fig. 16b), the location of three minima of the $\tau_{\mathrm{w}} / \tau_{\mathrm{y}}$ curves is found to be exactly at the static rigid zones on the cylinder's surface.

\section{CONCLUSION}

Flow of a thixotropic Bingham fluid over a circular cylinder in stationary and non-stationary laminar regimes has been investigated using a numerical approach. Re was in the range of 20-100 and Bn of $0.5-10$.

At $\operatorname{Re}=60$, the flow was found to be stationary at a $\mathrm{Bn}$ as small as 0.5 ; at which flow separation took place forming a pair of symmetrical recirculation zones behind the cylinder. At higher $\mathrm{Bn}$, i.e., $\mathrm{Bn} \geq 1$, a large static zone was instead observed at the rear of the cylinder; it could be considered as an extended cylinder.

At $\mathrm{Re}=100$ and $\mathrm{Bn} \leq 1$, the flow was non-stationary with vortex shedding behind the cylinder; no static rigid zones were observed on the cylinder's surface. The onset of the static zones was found at $\mathrm{Bn}=2$ though the flow was still non-stationary. At higher $\mathrm{Bn}$, the flow became stationary with or without flow separation, and thus unyielded zones were observed. The drag coefficient $C_{d}$ of the non-stationary flow was found to decrease with the increasing Re, and approached that of an equivalent Newtonian one, e.g., at $\operatorname{Re}=150$. This could be caused by the fact that the thixotropic material near the cylinder and in the wake was well broken and yielded, making it behave like a Newtonian one. 

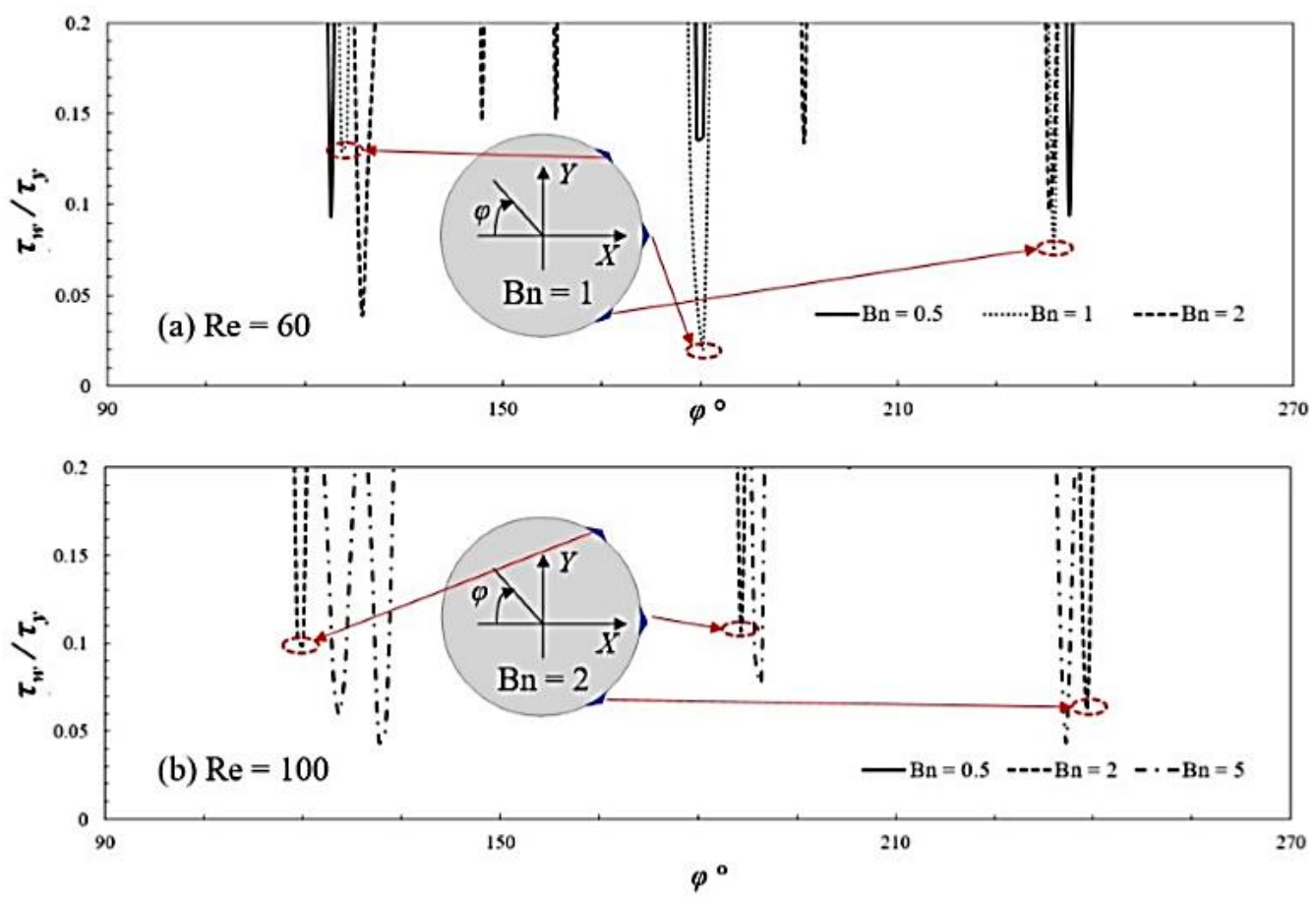

Fig. 17. Correlation between the minima of $\tau_{\mathrm{w}} / \tau_{\mathrm{y}}$ and the static rigid zones at $\operatorname{Re}=60$ and $\mathrm{Bn}=1$ (a), and $\operatorname{Re}=100$ and $B n=2(b)$.

An increase in Bn made the flow more stable, leading to a greater $C_{d}$. Indeed, $C_{d}$ was found to be a linear function of $\mathrm{Bn}$ in the range of $\mathrm{Bn}=0.5-5$, and at $\mathrm{Re}$ $=45,60$ and 100 .

Further study on the behaviors of the thixotropic and non-thixotropic Bingham flows in the non-stationary regime at higher Re could be carried out in the future.

\section{ACKNOWLEDGMENTS}

This research is funded by Ho Chi Minh City's Department of Science and Technology (HCMCDOST) and Institute for Computational Science and Technology (ICST) under grant number 08/2018//HD-KHCNTT.

\section{REFERENCES}

Barnes, H. A. (1997). Thixotropy-a review. Journal of Non-Newtonian Fluid Mechanics 70, $1-33$.

Braza, M., P. H. H. M. Chassaing and H. H. Minh (1986). Numerical study and physical analysis of the pressure and velocity fields in the near wake of a circular cylinder. Journal of fluid Mechanics 165, 79-130.

Bui, C. M. and T. X. Ho (2019). Numerical study of an unsteady flow of thixotropic liquids past a cylinder. AIP Advances 9(11), 115002.

Derksen, J. J. (2011). Simulations of thixotropic liquids. Applied Mathematical Modelling 35, $1656-1665$.
Mewis, J. and N. Wagner (2009). Thixotropy. Advances in Colloid and Interface Science 147, 214-227.

Moore, F. (1959). The rheology of ceramic slip and bodies. Transactions of the British Ceramic Society 58, 472-490.

Mossaz, S., P. Jay and A. Magnin (2012a). Experimental study of stationary inertial flows of a yield-stress fluid around a cylinder. Journal of Non-Newtonian Fluid Mechanics 189-190, $40-52$.

Mossaz, S., P. Jay and A. Magnin (2012b). Nonrecirculating and recirculating inertial flows of a viscoplastic fluid around a cylinder. Journal of Non-Newtonian Fluid Mechanics 177, 6475 .

Mossaz, S., P. Jay, O. Palsson and A. Magnin (2010). Criteria for the appearance of re-circulating and non-stationary regimes be-hind a cylinder in a viscoplastic fluid. Journal of Non-Newtonian Fluid Mechanics 165, 1525-1535.

Pantokratoras, A. (2018). Flow past a rotating sphere in a non-newtonian, power-law fluid, up to a reynolds number of 10,000. Chemical Engineering Science 181, 311 - 314.

Papanastasiou, T. C. (1987). Flows of materials with yield. Journal of Rheology 31, 385.

Park, J., K. Kwon and H. Choi (1998). Numerical solutions of flow past a circular cylinder at reynolds numbers up to 160 . KSME international Journal 12, 1200-1205. 
C. M. Bui and T. X. Ho / JAFM, Vol. 13, No. 5, pp. 1527-1538, 2020.

Ribeiro, V. M., P. M. Coelho, F. T. Pinho and M. A. Alves (2014). Viscoelastic fluid flow past a confined cylinder: Three-dimensional effects and stability. Chemical Engineering Science 11, 364-380.

Silva, A. L. E., A. Silveira-Neto and J. J. R. Damasceno (2003). Numerical simulation of two-dimensional flows over a circular cylinder using the immersed boundary method. Journal of Computational Physics 189, 351-370.

Singh, S. P. and S. Mittal (2005). Flow past a cylinder: shear layer instability and drag crisis. International Journal for Numerical Methods in Fluids 47, 75-98.

Syrakos, A., G. Georgiou and A. Alexandrou (2017). Thixotropic flow past a cylinder. Journal of Non-Newtonian Fluid Mechanics 220, 44-56.

Tokpavi, D. L., A. Magnin and P. Jay (2008). Very slow flow of bingham viscoplastic fluid around a circular cylinder. Journal of Non-Newtonian Fluid Mechanics 154, 65-76.

Tokpavi, D. L., P. Jay, A. Magnin and L. Jossic (2009). Experimental study of the very slow flow of a yield stress fluid around a circular cylinder. Journal of Non-Newtonian Fluid Mechanics 164, 35-44.

Tritton, D. J. (1959). Experiments on the flow past a circular cylinder at low Reynolds numbers. Journal of Fluid Mechanics 6, 547-567.

Wang, Z., W. Lou, B. Sun, S. Pan, X. Zhao and H. Liu (2019). A model for predicting bubble velocity in yield stress fluid at low Reynolds number. Chemical Engineering Science 201, $325-338$.

Williamson, C. H. (1996). Vortex dynamics in the cylinder wake. Annual review of fluid mechanics 28, 477-539. 